\title{
Aberrant static and dynamic functional connectivity of the executive control network in lung cancer patients after chemotherapy: a longitudinal fMRI study
}

\author{
Lanyue Hu ${ }^{1} \cdot$ Huiyou Chen ${ }^{1} \cdot$ Wen Su ${ }^{1} \cdot$ Yujie Zhang ${ }^{1} \cdot$ Jia You ${ }^{1} \cdot$ Wei Gu ${ }^{2} \cdot$ Zhenyu Xiong $^{3} \cdot$ Xindao Yin $^{1}$. \\ Yu-Chen Chen ${ }^{1}$
}

Published online: 17 April 2020

(C) The Author(s) 2020

\begin{abstract}
Objective The purpose of the current study was to investigate chemotherapy-related variations in the intrinsic static and dynamic functional connectivity ( $\mathrm{sFC}$ and $\mathrm{dFC}$, respectively) of the executive control network (ECN) in lung cancer patients.

Materials and methods In this study, we evaluated 18 lung cancer patients scanned before and after adjuvant chemotherapy treatment and compared the patients with 21 healthy controls (HCs). All subjects underwent resting-state functional MRI (rsfMRI). We constructed the sFC and dFC of the bilateral dorsolateral prefrontal cortex (DLPFC) using a sliding-window approach, and the correlations between the changed sFC or dFC and cognitive performance were analyzed.

Results Whole-brain sFC analysis showed that the lung cancer patients showed significant FC pattern changes in the bilateral DLPFC, mainly in the bilateral superior frontal gyrus (SFG), bilateral middle frontal gyrus, left superior temporal gyrus, left inferior parietal lobe and the right insula. Furthermore, after chemotherapy, the lung cancer patients showed significantly reduced $\mathrm{dFC}$ variability between the right DLPFC and right precuneus compared with HCs. In addition, the decreased dFC between the right DLPFC and left SFG in the lung cancer patients after chemotherapy in state 1 and between the right DLPFC and left insula in the lung cancer patients before chemotherapy in state 2 were negatively correlated with MoCA $\operatorname{scores}((\mathrm{r}=-0.520, p=0.039 ; \mathrm{r}$ $=-0.548, p=0.028$, respectively).

Conclusions Our results reveal that dynamic connectivity analysis is more effective and sensitive than methods that assume static brain states for linking brain FC patterns and chemotherapy.
\end{abstract}

Keywords Lung cancer $\cdot$ Static connectivity $\cdot$ Dynamic connectivity $\cdot$ Executive control network $\cdot$ Resting-state fMRI

\section{Introduction}

Accompanying the increasing morbidity (ranking first among globally diagnosed cancers (Bray et al. 2018)) and overall survival rate of lung cancer, chemotherapy-related cognitive

Lanyue $\mathrm{Hu}$ and Huiyou Chen contributed equally to this work.

Yu-Chen Chen

chenyuchen1989@126.com

1 Department of Radiology, Nanjing First Hospital, Nanjing Medical University, No.68, Changle Road, Nanjing 210006, China

2 Department of Respiratory Medicine, Nanjing First Hospital, Nanjing Medical University, Nanjing, China

3 Department of Radiation Oncology, The University of Texas Southwestern Medical Center, Dallas, TX, USA impairment (CRCI) in lung cancer has become a wellrecognized side effect during and after adjuvant chemotherapy treatment (Janelsins et al. 2018). Platinum-based chemotherapy was a broadly used global standard of care for advanced lung cancer(Ettinger et al. 2017; Scagliotti et al. 2008). Kaasa et al. (1988) indicated that non-small cell lung cancer patients exhibited cognitive deficits as soon as one month after adjuvant chemotherapy treatment. Longitudinal neuropsychological assessment research studies (Janelsins et al. 2011) suggest that up to $75 \%$ of cancer patients experience multiple-domain CRCI, particularly impairments in executive functions, attention, memory and processing speed, during treatment and up to $35 \%$ experience persistent CRCI for months or years following treatment completion (Grosshans et al. 2008; Janelsins et al. 2014; Li and Caeyenberghs 2018; Mandelblatt et al. 2013; Pergolizzi et al. 2019). As a major component of advanced cognitive functions, executive functions are vital to 
human autonomy and are a major determinant of problem behaviors and disabilities (Royall et al. 2002) that incorporate and command more basic cognitive processes (Baddeley 2000; Funahashi and Andreau 2013; Jodzio and Biechowska 2010). Improving our understanding of the changes that occur in executive function-related neurological mechanisms in lung cancer patients receiving adjuvant chemotherapy might be conducive to adjusting treatment regimens appropriately and achieving better functional improvements.

Remarkably, resting-state functional MRI (rs-fMRI) has been proved as a powerful noninvasive method for examining the brain functional network. rs-fMRI evaluates the temporal correlation of intrinsic low-frequency fluctuations based on blood oxygenation level-dependent (BOLD) signals between brain regions during rest(Rosazza and Minati 2011). In practice, using this technique, functional connectivity studies have reported a number of resting-state networks (RSNs) that consist of anatomically separated, but functionally connected regions displaying a high level of correlated BOLD signal activity(van den Heuvel et al. 2008; Van Dijk et al. 2010), and the corroborated executive control network (ECN) mainly involves the dorsolateral prefrontal cortex (DLPFC), dorsal medial prefrontal cortex (DMPFC) and posterior parietal cortex (PPC) (Bressler and Menon 2010; Shirer et al. 2012; Weiland et al. 2014). Recently, a neuroimaging community report showing the altered FC of the ECN in cancer patients during and after chemotherapy has gained increasing attention. Piccirillo et al. (Piccirillo et al. 2015) focused on the FC changes in the frontoparietal network and the cinguloopercular executive control network in breast cancer patients using a case-control design. Their findings indicated that the standard therapeutic levels of chemotherapy in breast cancer resulted in disrupted FC in the brain networks supporting attention and executive control function. Furthermore, Shelli and colleagues (Kesler et al. 2011) examined differences in prefrontal regions between breast cancer patients received chemotherapy or not and healthy controls. The findings demonstrated hypoactivation in the left medial dorsolateral prefrontal lobe and premotor cortex in breast cancer patients received chemotherapy compared with the healthy controls.

Nevertheless, the aforementioned studies on the FC patterns of the ECN in cancer patients after chemotherapy assumed that FC was stationary during the process of scanning, which is referred to as static FC ( $\mathrm{sFC}$ ). Studies have displayed that the resting brain is an extremely dynamic system and that the functional connectivity states of human brain vary over time (Allen et al. 2014; Hutchison et al. 2013). Thus, the $\mathrm{sFC}$ approach has distinct limitations in reflecting dynamic brain processes. Dynamic functional connectivity (dFC) analysis overcomes this static restriction and can reflect the timevarying patterns and temporal dynamic characteristics of $\mathrm{FC}$ (Hutchison et al. 2013). Studies have shown that dFC analysis produces more sensitive results than $\mathrm{sFC}$ analysis for identifying group differences between healthy controls and patients with various neurological diseases (Chen et al. 2018; Fu et al. 2018; Yang et al. 2019). Notably, a previous study (Kesler et al. 2017) demonstrated that functional dynamics were distinctly lower in breast cancer patients than in control participants.

Previous studies on CRCI in lung cancer have mainly concentrated on brain structure changes and default mode network disruption (Bromis et al. 2017; Simo et al. 2015, 2016, 2018; Welzel et al. 2008). Bromis et al. (2017) found significant FC disruptions within all the rest-state networks of lung cancer patients after chemotherapy compared with the healthy controls. Additionally, other results suggest that the reduced rest-state FC pattern within the default mode network (DMN) and the cognitive disorder were associated with cancer and chemotherapy in lung cancer patients (Simo et al. 2018; Zhang et al. 2019). Unfortunately, executive function disorders related to cancer and chemotherapy in the lung cancer patient population remain poorly understood, and further explorations are urgently necessary.

The present study aimed to investigate cancer- and chemotherapy-related intrinsic $\mathrm{SFC}$ and $\mathrm{dFC}$ differences within the ECN in rs-fMRI employing the seed-based approach in lung cancer survivors. The prefrontal cortex has been demonstrated to be an important structure for executive functions(Funahashi and Andreau 2013). Moreover, the DLPFC, in the middle frontal gyrus, which is the core node of the ECN, is involved in functions including working memory, prospective memory and executive function (Mitchell et al. 2008; Pochon et al. 2001). Based on previous neuroimaging findings, we selected the bilateral DLPFC as the critical seed region and hypothesized that (1) $\mathrm{SFC}$ and $\mathrm{dFC}$ patterns in the DLPFC are significantly functionally disrupted in lung cancer patients after chemotherapy and (2) that altered $\mathrm{sFC}$ or $\mathrm{dFC}$ was associated with cognitive performance in lung cancer patients. As far as we know, this is the first longitudinal study to explore links between $\mathrm{sFC}$ and $\mathrm{dFC}$ changes in $\mathrm{ECN}$ patterns and chemotherapy in the lung cancer patient population.

\section{Materials and methods}

\section{Participants}

The prospective study was approved by the Research Ethics Committee of Nanjing Medical University, and all subjects signed informed consent before their participation in the study protocol.

This was a longitudinal study of lung cancer patients scheduled to incorporate scans at baseline $(\mathrm{t} 0)$ and three to six months (t1) after receiving adjuvant chemotherapy and to include age/sex-matched healthy controls (HCs). Eighteen lung cancer patients from the Department of Respiratory Medicine, 
Nanjing First Hospital and 21 healthy controls (aged between 48 and 70 years, received at least 6 years of education) were enrolled through online advertisements and age, sex, and education level-matched between May 2018 and August 2019. All participants were right-handed. Among the patients treated with chemotherapy (platinum-based doublet with pemetrexed), 10 patients received cisplatin-based therapy, and 8 patients received carboplatin-based therapy for three to six months. No participants were excluded from the fMRI analysis because of excessive head motion during scanning. The exclusion criteria for all participants were: receipt prophylactic cranial irradiation; presence of a metastatic brain tumor; declared history of known stroke, craniocerebral trauma, epilepsy, Alzheimer's disease, Parkinson's disease, other acute psychiatric or neurological illnesses; presence of a major medical illness (e.g., anemia, severe heart diseases, thyroid dysfunction or abnormality in liver or kidney function); and presence of severe vision or hearing loss. The neuropsychological status and general cognitive function of the participants were established using the Mini Mental State Exam (MMSE) (Galea and Woodward 2005) and Montreal Cognitive Assessment (MoCA) (Nasreddine et al. 2005).

\section{MRI acquisition}

All MRI data were acquired in 3.0 T MRI scanner (Ingenia, Philips Medical Systems, Netherlands) with an 8-channel receiver array head coil and parallel imaging was employed. Tight but comfortable foam padding was used to minimize head motion, and earplugs were used to alleviate scanner noise. Subjects were instructed to lie with their eyes closed and stay awake, to not thinking things in particular. For each participant, routine MRI sequences, including thick-slice T2and T1-weighted imaging as well as T2 fluid-attenuated inversion recovery (FLAIR) imaging, were performed to ensure that there were no visible brain lesions or brain metastases.

The resting state fMRI data were obtained with the gradient echo-planar imaging sequence as follows: repetition time $(\mathrm{TR})=2000 \mathrm{~ms}$; echo time $(\mathrm{TE})=30 \mathrm{~ms}$; slices $=36$; thickness $=4 \mathrm{~mm}$; gap $=0 \mathrm{~mm}$; field of view $(F O V)=240 \mathrm{~mm} \times$ $240 \mathrm{~mm}$; acquisition matrix $=64 \times 64$; and flip angle $(\mathrm{FA})=$ $90^{\circ}$. High-resolution three-dimensional T1-weighted images (3D-T1WI) data were acquired with magnetization-prepared rapid gradient-echo sequence as follows: $\mathrm{TR}=8.1 \mathrm{~ms}$; $\mathrm{TE}=$ $3.7 \mathrm{~ms}$; slices $=170$; thickness $=1 \mathrm{~mm}$; gap $=0 \mathrm{~mm} ; \mathrm{FA}=8^{\circ}$; acquisition matrix $=256 \times 256$; and $\mathrm{FOV}=256 \mathrm{~mm} \times 256$ $\mathrm{mm}$. The resting state fMRI sequence lasted $488 \mathrm{~s}$, and the structural sequence lasted $329 \mathrm{~s}$.

\section{Data preprocessing}

Functional preprocessing was performed using Statistical Parametric Mapping (SPM8; http://www.fil.ion.ucl.ac.uk/ spm) and the Graph Theoretical Network Analysis Toolbox for Imaging Connectomics (GRETNA) (Wang et al. 2015) (2. 0.0A http://www.nitrc.org/projects/gretna/). The processing pipeline included the following stages: (i) The first $10 \mathrm{vol}-$ umes were discarded to calculate the time required for participants to adapt to the scanning environment. (ii) Slice timing corrected and realigned were performed for the remaining 220 images, and head motion $>2.0-\mathrm{mm}$ in each direction or rotation angle $>2.0^{\circ}$ were removed from analysis. (iii) The remaining dataset was normalized to the Montreal Neurological Institute (MNI) EPI template (reslicing voxel size as $3 \times 3 \mathrm{~mm}^{3}$ ). (iv) Spatially smoothing with a Gaussian kernel $[6 \mathrm{~mm}$ full width at half maximum (FWHM)]. (v) Detrending and filtering $(0.01-0.08 \mathrm{~Hz})$ were performed in turn. Subsequently, several nuisance signals including head motion, the global mean, and signals from white matter (WM) and the cerebrospinal fluid (CSF) were regressed from the data.

\section{Static FC analysis}

The seed regions in the bilateral DLPFC were extracted from the Brodmann template using WFU Pick Atlas software (Maldjian et al. 2003). For sFC analysis, the mean time series of the signal in the bilateral DLPFC were obtained to serve as the seed time course, and the Pearson correlation coefficients (r) were finally calculated between the mean time series of each seed region and all brain voxel time series. Finally, a Fisher's z-transform was used to obtain variables approximating a normal distribution (Lowe et al. 1998).

\section{Dynamic FC analysis}

Dynamic FC analysis was performed using the Dynamic Brain Connectome (Dynamic BC) toolbox (Liao et al. 2014) (V2.1 http://restfmri.net/forum/DynamicBC). Temporal dynamic patterns were characterized by using a sliding-window approach, which was created by convolving a rectangle with a Gaussian kernel $(\sigma=3 \mathrm{TR})$. Previous studies have revealed that a frequency interval of $[0-1 / \mathrm{w}] \mathrm{Hz}$ should be the target due to the low-pass filtering effect of the window and that the minimum window length should be above $1 / \mathrm{f}_{\min }$ (Leonardi and Van De Ville 2015). Some researchers have applied a slidingwindow length of as small as $10 \mathrm{~s}$ (Thompson et al. 2013) and as long as $180 \mathrm{~s}$ (Gonzalez-Castillo et al. 2015). Thus, the window size was chosen to be 20 TRs ( $40 \mathrm{~s}$ ), and the window overlap was selected to be $95 \%$ (window-shifting step size of 1 $\mathrm{TR}$ ), resulting in $\mathrm{W}=201$ windows (Chen et al. 2017). The temporal correlation coefficient between the time courses of each seed and that for the other brain voxels was calculated for each sliding window. For each subject, a range of slidingwindow correlation maps was acquired. To characterize the time variability in $\mathrm{FC}$, the standard deviation of each voxel 
across a number of windows was calculated and the Fisher Ztransformed used to acquire variables similar to a normal distribution (Liao et al. 2018).

\section{Clustering analysis}

To assess reoccurring dFC patterns, a k-means clustering algorithm was used to analyze dFC estimates of all subjects (combining the lung cancer and HC groups) (Allen et al. 2014). We used the L1 distance function to assess the similarity between sliding window FCs, as L1 distance has been proved to be an effective measurement method for highdimensional data (Aggarwal et al. 2001; Allen et al. 2014). We determined the number of clusters to be three using the elbow criterion of the cluster validity index, which is computed as the ratio between the within-cluster distance and between-cluster distance. The gained clustering centroids were then served as the departure points to cluster all $\mathrm{dFC}$ windows from all subjects.

\section{Statistical analysis}

SPSS software (version 19.0; SPSS, Chicago, IL) was used for statistical analyses, and a corrected statistically significance level of $\mathrm{p}<0.05$ was obtained. Group comparisons of demographic information or clinical measures were performed using two-sample t-tests to compare continuous data between the lung cancer groups and HC group.

A one-sample t-test was performed to analyze individual sFC maps in a voxel wise manner. The test was used to determine the sFC patterns in regions with significant connectivity to the specific seeds in each group. Two-sample t-tests were then performed to determine the $\mathrm{sFC}$ and $\mathrm{dFC}$ differences between $\mathrm{HCs}$ and lung cancer patients at $\mathrm{t} 0$ and $\mathrm{t} 1$, and paired t-tests were used to estimate the $\mathrm{sFC}$ and $\mathrm{dFC}$ differences between $\mathrm{t} 0$ and $\mathrm{t} 1$ in the lung cancer patients. Correction was implemented using false discovery rate (FDR) approach and significance threshold was set at $p<0.01$. Age, sex and education were used as nuisance covariates to control for the effects of these factors on the results.

In order to study the temporal properties of $\mathrm{dFC}$ patterns, we calculated some dynamic indicators including the mean dwell time (MDT) and the number of transitions (NT). The average time (in windows) spent in a state before transitioning to another state was defined as the MDT. In addition, the NT stands for the number of transitions between states. Group differences tests in the MDT and NT between HCs and lung cancer patients at $\mathrm{t} 0$ and $\mathrm{t} 1$ were checked using a two-sample $\mathrm{t}$ test ( $\mathrm{P}<0.01$, FDR correction). Between-group differences among the lung cancer patients at $\mathrm{t} 0$ and $\mathrm{t} 1$ were investigated using paired t-tests ( $\mathrm{P}<0.01$, FDR correction).

To evaluate the correlational relationship of abnormal $\mathrm{sFC}$ or $\mathrm{dFC}$ and cognitive performance, Pearson correlation analyses were implemented by using SPSS19.0. P less than 0.05 was considered statistically significant. Partial correlations were calculated after correcting for age, sex and education.

\section{Results}

\section{Demographic and neuropsychological data}

All the detailed demographics and a summary of the histological diagnosis and tumor stage are included in Table 1. There was no significant difference in age, sex, education level, or MMSE score between the lung cancer patients and the HCs (all $\mathrm{p}>0.05$ ). The lung cancer patient group at $\mathrm{t} 1 \mathrm{had}$ worse MoCA scores than the HC group and lung cancer patient group at $\mathrm{t} 0(\mathrm{p}<0.001)$.

\section{Static FC results}

\section{Within-group comparisons}

Individual sFC maps for each group are shown in Fig. 1. The bilateral DLPFC mainly displayed positive FC within the ECN regions, mainly involving the prefrontal cortex (PFC), PPC, the temporal cortex, and some subcortical regions, in HCs (Fig. 1A) and lung cancer patients at t0 and t1 (Fig. 1C and E).

\section{Between-group comparisons}

Seed-based whole-brain sFC analysis revealed the sFC patterns of lung cancer patients and HCs (Table 2; Figs. 1 and 2). Based on the seeds of the bilateral DLPFC, relative to the $\mathrm{HCs}$, the lung cancer patients at $\mathrm{t} 0$ revealed marked decreased sFC between the right DLPFC and left inferior parietal lobule (IPL) or left superior temporal gyrus (STG) and between the left DLPFC and left IPL; the lung cancer patients at t1 showed significant reductions in $\mathrm{sFC}$ between the right DLPFC and bilateral SFG, left middle frontal gyrus (MFG), or right medial cingulate gyrus (MCG) and between the left DLPFC and right MFG. Compared with those at $\mathrm{t} 0$, the lung cancer patients at $\mathrm{t} 1$ showed decreased sFC between the right DLPFC and left SFG and increased sFC between the left DLPFC and right insula.

\section{Dynamic FC results}

\section{Dynamic FC variability}

The differences in $\mathrm{dFC}$ variability between the lung cancer patient groups and the $\mathrm{HC}$ group are illustrated in Fig. 3; Table 3. Relative to the $\mathrm{HCs}$, the lung cancer patients at $\mathrm{t} 0$ 
Table 1 Demographic and clinical characteristics of all subjects

\begin{tabular}{|c|c|c|c|c|}
\hline & \multicolumn{2}{|c|}{ Lung cancer $(\mathrm{n}=18)$} & HCs $(n=21)$ & $p$ value \\
\hline Age, year & \multicolumn{2}{|l|}{$59.72 \pm 9.04$} & $58.57 \pm 9.61$ & $0.703^{\mathrm{a}}$ \\
\hline Education, year & \multicolumn{2}{|l|}{$10.59 \pm 4.25$} & $10.71 \pm 1.88$ & $0.904^{\mathrm{a}}$ \\
\hline Gender, male/female & \multicolumn{2}{|l|}{$13 / 5$} & $13 / 8$ & $0.828^{b}$ \\
\hline \multicolumn{5}{|l|}{ Histological diagnosis } \\
\hline Adenocarcinoma & \multicolumn{2}{|l|}{$9(50)$} & & \\
\hline Squamous cell carcinoma & \multicolumn{2}{|l|}{$7(39)$} & & \\
\hline SCLC & \multicolumn{2}{|l|}{$2(11)$} & & \\
\hline \multicolumn{5}{|l|}{ Tumor stage } \\
\hline Limited disease & \multicolumn{2}{|l|}{$1(5)$} & & \\
\hline Extensive disease & \multicolumn{2}{|l|}{$1(5)$} & & \\
\hline I & \multicolumn{2}{|l|}{$3(17)$} & & \\
\hline II & \multicolumn{2}{|l|}{$5(28)$} & & \\
\hline III & \multicolumn{2}{|l|}{$4(22)$} & & \\
\hline \multirow[t]{2}{*}{ IV } & \multicolumn{2}{|l|}{$4(22)$} & & \\
\hline & t0 & $\mathrm{t} 1$ & & \\
\hline MMSE & $26.78 \pm 1.17$ & $26.61 \pm 1.19$ & $27.19 \pm 1.03$ & $0.248^{\mathrm{a} 1} ; 0.163^{\mathrm{a} 2} ; 0.698^{\mathrm{a} 3}$ \\
\hline $\mathrm{MoCA}$ & $22.22 \pm 1.93$ & $22.06 \pm 2.94$ & $25.76 \pm 1.61$ & $0.000^{* \mathrm{a} 1} ; 0.000^{* \mathrm{a} 2} ; 0.817^{\mathrm{a} 3}$ \\
\hline
\end{tabular}

Data are expressed as Mean \pm SD, $\mathrm{n}(\%)$ or median (range), ${ }^{\mathrm{a}}$ The $\mathrm{P}$ values are obtained by using two sample t-test; ${ }^{\mathrm{b}}$ The $\mathrm{P}$ values are obtained by using $\chi 2$ test. ${ }^{*} P<0.05$ is considered significant. ${ }^{\text {al }}$ The $\mathrm{P}$ value between lung cancer patients before chemotherapy and $\mathrm{HCs} ;{ }^{\mathrm{a} 2}$ The $\mathrm{P}$ value between lung cancer patients after chemotherapy and $\mathrm{HCs} ;{ }^{\mathrm{a} 3}$ The $\mathrm{P}$ value between lung cancer patients before and after chemotherapy. MMSE, Mini Mental State Exam; MoCA, Montreal Cognitive Assessment; HCs, healthy controls exhibited significantly increased $\mathrm{dFC}$ variability between the bilateral DLPFC and left precuneus; at t1, significantly reduced $\mathrm{dFC}$ variability between the right DLPFC and right superior parietal lobule (SPL) and between the left DLPFC and left SFG was revealed. In addition, paired t-tests showed the lung cancer patients at $\mathrm{t} 1$ exhibited significantly decreased $\mathrm{dFC}$ variability between the right DLPFC and right precuneus, the left DLPFC and right inferior frontal gyrus (IFG) or left inferior temporal gyrus (ITG).

\section{Dynamic connectivity states and the associated brain dynamic FC}

Figure 4 shows the transition matrices of different states, which were extracted using the K-means clustering method with a cluster size of 3 . The different FCs between groups in each state are displayed in Figs. 5 and 6; Tables 4 and 5.

Based on the seeds of the bilateral DLPFC, in state 1, compared with the $\mathrm{HCs}$, the lung cancer patients at $\mathrm{t} 0$ showed significantly decreased dFC between the right DLPFC and the left fusiform and between the left DLPFC and left inferior orbitofrontal gyrus; the lung cancer patients at $\mathrm{t} 1$ showed significantly increased dFC between the right DLPFC and the left posterior cingulate cortex (PCC) and decreased $\mathrm{dFC}$ between the left DLPFC and triangle inferior frontal gyrus (IFG).
Compared with those at $\mathrm{t} 0$, the lung cancer patients at $\mathrm{t} 1 \mathrm{ex}-$ hibited significantly decreased dFC between the right DLPFC and the left IFG and between the left DLPFC and right superior orbit frontal gyrus. In state 2, relative to the $\mathrm{HCs}$, the lung cancer patients at t0 showed significantly decreased $\mathrm{dFC}$ between the right DLPFC and left insula or MFG and between the left DLPFC and right MCG; at t1, the lung cancer patients showed significantly reduced $\mathrm{dFC}$ between the right DLPFC and left thalamus. Compared with those at $\mathrm{t} 0$, the lung cancer patients at $\mathrm{t} 1$ showed significantly increased $\mathrm{dFC}$ between the right DLPFC and left insula or left MFG and decreased dFC between the left DLPFC and right MFG. In state 3, the lung cancer patients at $\mathrm{t} 1$ showed significantly increased $\mathrm{dFC}$ between the left DLPFC and right MCG compared with those at t0 and the HCs.

Based on the seed of the right DLPFC, in regard to the between-group comparisons of the MDT and NT in each state, we have found that the lung cancer patients at 0 seemed to stay in state 1 for a shorter time ( $\mathrm{t}=-2.045, p=0.048)$ and that the lung cancer patients at both $\mathrm{t} 0$ and $\mathrm{t} 1$ appeared to have a lower NT $(\mathrm{t}=-$ 2.274, $p=0.029$; $\mathrm{t}=-3.329, p=0.003$ ) than the HCs (Fig. 7A, B).

Based on the seed of the left DLPFC, for the between-group comparisons of the MDT and NT in each state, we found that the lung cancer patients after chemotherapy seemed to stay in state 3 for a shorter time than the $\operatorname{HCs}(\mathrm{t}=-2.292, p=00,029)$, and paired t-tests showed that the lung cancer patients at $\mathrm{t} 1 \mathrm{had}$ a lower NT than those at $\mathrm{t} 0(\mathrm{t}=-2.233, p=0.039)($ Fig. $7 \mathrm{C}, \mathrm{D})$. 
Fig. 1 A, C, E Visualization of significant $F C$ patterns of the right DLPFC by whole-brain scanning using a one-sample t-test to compare HCs and lung cancer patients at $\mathrm{t} 0$ and $\mathrm{t} 1$. B Compared with $\mathrm{HCs}$, lung cancer patients at t0 showed decreased FC between the R-DLPFC and left STG or left IPL. D Compared with the HCs, the lung cancer patients at $\mathrm{t} 1 \mathrm{ex}-$ hibited reduced $\mathrm{FC}$ between the R-DLPFC and bilateral SFG, left MFG, or right MCG. F Compared with those at $\mathrm{t} 0$, the lung cancer patients at $\mathrm{t} 1$ showed reduced FC between the R-DLPFC and right MFG or left SFG. Significance thresholds were corrected using an FDR criterion and set at $\mathrm{p}<$ 0.01 . HCs: healthy controls; t0: lung cancer patients before chemotherapy; t1: lung cancer patients after chemotherapy

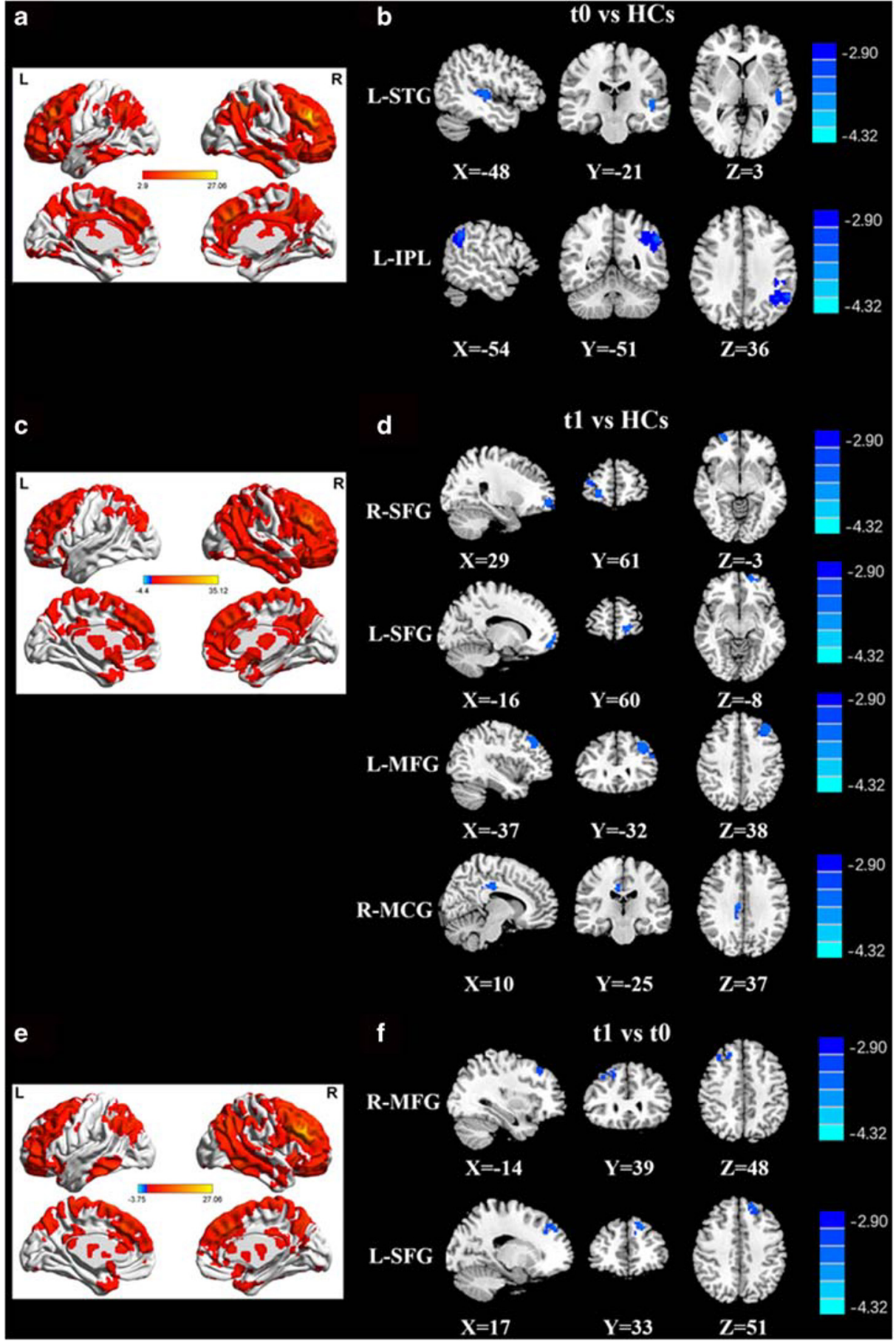

\section{Correlation analysis results}

There were no significant correlational relationships between $\mathrm{sFC}$ and MoCA scores at $\mathrm{t} 0$ or $\mathrm{t} 1$ or in the HCs. In state 1 , in comparisons within the lung cancer groups at $\mathrm{t} 1$ versus $\mathrm{t} 0$, the decreased dFC of the right DLPFC to the left SFG was negatively correlated with MoCA scores $(\mathrm{r}=-0.520, p=0.039)$ (Fig. 8A). Moreover, in state 2, compared with that in the $\mathrm{HCs}$, the decreased $\mathrm{dFC}$ of the right DLPFC to the left insula in the lung cancer group at $\mathrm{t} 0$ was negatively associated with reduced MoCA scores ( $\mathrm{r}=-0.548, p=0.028)$ (Fig. 8B).

\section{Discussion}

CRCI has been quantified using neuropsychological test batteries, and medical imaging research using rs-fMRI has 
Fig. 2 A, C, E Significant FC patterns in the left DLPFC determined by whole-brain scanning using a one-sample t-test to compare healthy controls and lung cancer patients at 0 or $\mathrm{t} 1$. B Compared with the HCs, the lung cancer patients at 0 showed decreased FC between the LDLPFC and left IPL. D

Compared with the healthy controls, the lung cancer patients at $\mathrm{t} 1$ exhibited reduced FC between the L-DLPFC and right MFG. F Compared with those at $\mathrm{t} 0$, the lung cancer patients at $\mathrm{t} 1$ showed increased FC between the LDLPFC and right insula. Significance thresholds were corrected using an FDR criterion and set at $\mathrm{p}<0.01$. HCs: healthy controls; t0: lung cancer patients before chemotherapy; $\mathrm{t} 1$ : lung cancer patients after chemotherapy

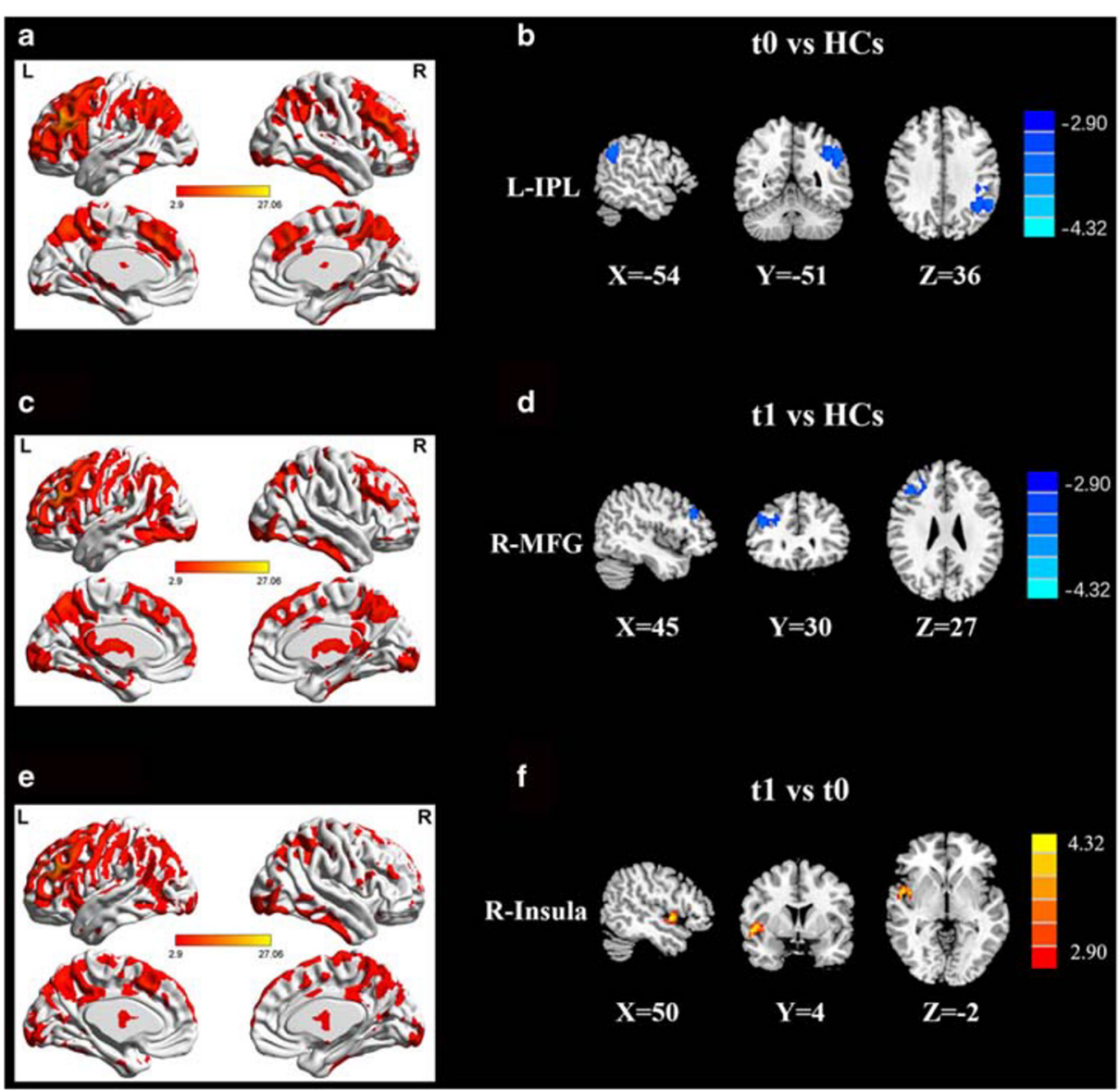

attempted to elucidate the underlying neurobiological mechanisms. Indeed, research on rest-state $\mathrm{sFC}$ changes related to chemotherapy has witnessed exponential growth. In the current study, we found that the regions interacting with the bilateral DLPFC were mainly distributed in the PFC, partial parietal and temporal lobe. The DLPFC, which is the core node of the ECN, is involved in functions including working memory, prospective memory and executive function (Barbey et al. 2013; Pochon et al. 2001). The PFC and its cortical and subcortical connections play a central role in processing
Table 2 Abnormal static functional connectivity between groups

\begin{tabular}{|c|c|c|c|c|c|c|}
\hline ROIs & Group comparisons & Brain region & BA & $\begin{array}{l}\text { MNI Coordinates } \\
\mathrm{x}, \mathrm{y}, \mathrm{z}(\mathrm{mm})\end{array}$ & T score & Voxels \\
\hline \multirow[t]{8}{*}{ R-DLPFC } & \multirow[t]{2}{*}{ t0 vs. HCs } & Temporal_Sup_L & 81 & $-48,-21,3$ & -4.2286 & 89 \\
\hline & & Parietal_Inf_L & 61 & $-54,-51,36$ & -5.8758 & 315 \\
\hline & \multirow[t]{4}{*}{ t1 vs. HCs } & Frontal_Sup_R & 6 & $24,57,-6$ & -4.4452 & 109 \\
\hline & & Frontal_Sup_L & 5 & $-15,60,-9$ & -4.1401 & 52 \\
\hline & & Frontal_Mid_L & 7 & $-39,30,42$ & -4.689 & 145 \\
\hline & & $\begin{array}{l}\text { Cingulum_Mid_ } \\
\mathrm{R}\end{array}$ & 34 & $9,-21,33$ & -4.1414 & 65 \\
\hline & \multirow[t]{2}{*}{ t1 vs. t0 } & Frontal_Sup_L & 3 & $-18,45,22$ & -4.5187 & 85 \\
\hline & & Frontal_Mid_R & 8 & $45,33,51$ & -3.7625 & 45 \\
\hline \multirow[t]{3}{*}{ L-DLPFC } & t0 vs. HCs & Parietal_Inf_L & 61 & $-54,-51,36$ & -4.907 & 315 \\
\hline & t1 vs. HCs & Frontal_Mid_R & 8 & $45,30,27$ & -3.8228 & 176 \\
\hline & t1 vs. t0 & Insula_R & 30 & $51,3,-3$ & 4.6003 & 84 \\
\hline
\end{tabular}

Thresholds were set at a corrected $\mathrm{p}<0.01$ corrected by FDR criterion. BA, Brodmann's area; MNI, Montreal Neurological Institute; L, left; R, right; $t 0$, lung cancer patients before chemotherapy; $t$, lung cancer patients after chemotherapy; HCs, healthy controls 


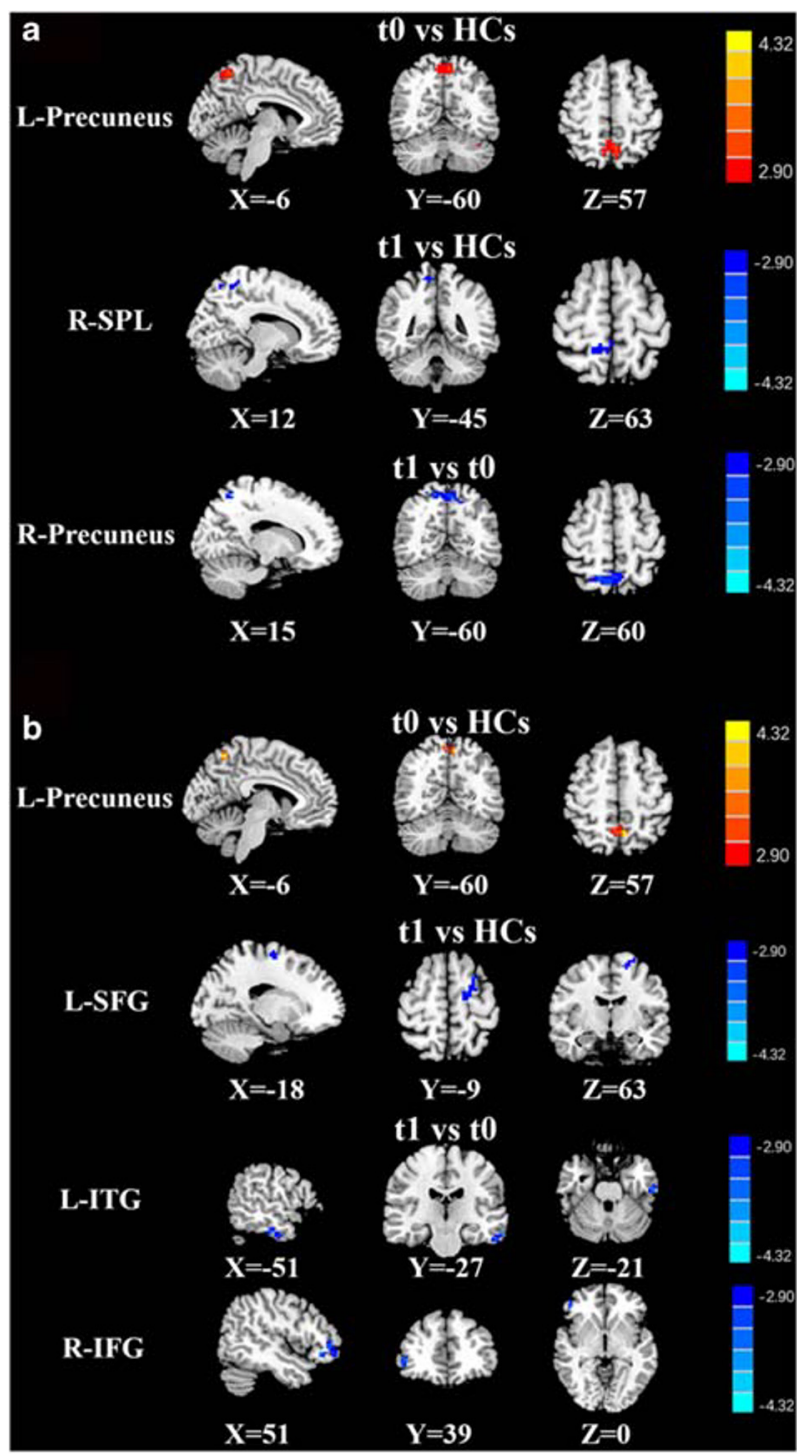

Fig. 3 Brain regions with significant intergroup differences in $\mathrm{dFC}$ variability. A Using the right DLPFC as the seed region, significant group differences were detected in multiple brain regions. B Using the left DLPFC as the seed region, significant group differences were detected in multiple brain regions. Significance thresholds were corrected using an FDR criterion and set at $\mathrm{p}<0.01$ executive functions (Rusnakova et al. 2011). In addition, the STG is considered to be a central brain region involved in processing executive functions together with frontal areas that form a cognitive network (Bockova et al. 2007). Recent studies have demonstrated that the PFC and STG are impaired in lung cancer patients after chemotherapy (McDonald et al. 2012; Simo et al. 2018; Wang et al. 2016). In breast cancer patients, reduced FC was chiefly seen between the right DLPFC and the right IFG, right MFG, and left STG compared with HCs (Wang et al. ). One month after standard chemotherapy, McDonald et al. (McDonald et al. ) reported that both patients receiving chemotherapy and those not requiring chemotherapy showed decreased activation of the IFG compared to HCs. Our findings are not only consistent with literature reports but are also unique. Our findings support the idea that the cancer disease process itself affects brain functioning (Menning et al. 2017); in addition, by means of longitudinal comparison, we confirmed the exact effect of chemotherapy on sFC.

Furthermore, the decreased sFC between the bilateral DLPFC and the left IPL was demonstrated in prechemotherapy lung cancer patients compared with HCs. Sanne et al. (Menning et al. 2017) detected that parietal activation decreased in a breast cancer group that was not exposed to any systemic treatment compared to an HC group. Based on previous studies, we speculated that the parietal areas are more susceptible to factors related to stressful events, such as cancer diagnosis and surgery. In contrast, the lung cancer group showed significantly increased sFC between the left DLPFC and right insula after chemotherapy. The insula is believed to be involved in emotion and consciousness. A previous study (Feng et al. 2019) reported that patients treated with chemotherapy showed persistent anxiety and depression symptoms over time and that psychological symptom score changes were significantly positively associated with FC changes between the left hippocampus and left insula. We hypothesized that the increased sFC between the left DLPFC and right insula may be a mechanism of selfregulating negative emotion in lung cancer patients.
Table 3 Abnormal dynamic functional connectivity between groups

\begin{tabular}{|c|c|c|c|c|c|c|}
\hline ROIs & Group comparisons & Brain region & $\mathrm{BA}$ & $\begin{array}{l}\text { MNI Coordinates } \\
\mathrm{x}, \mathrm{y}, \mathrm{z}(\mathrm{mm})\end{array}$ & T score & Voxels \\
\hline \multirow[t]{3}{*}{ R-DLPFC } & t0 vs. HCs & Precuneus_L & 67 & $-6,-60,57$ & 5.0394 & 77 \\
\hline & t1 vs. HCs & Parietal_Sup_R & 60 & $12,-45,63$ & -3.6612 & 43 \\
\hline & t1 vs. $\mathrm{t} 0$ & Precuneus_L & 67 & $15,-60,60$ & -5.686 & 116 \\
\hline \multirow[t]{4}{*}{ L-DLPFC } & t0 vs. HCs & Precuneus_L & 67 & $-6,-60,57$ & 5.0394 & 77 \\
\hline & t1 vs. HCs & Frontal_Sup_L & 3 & $-18-9,63$ & -4.2194 & 42 \\
\hline & t1 vs. t0 & $\begin{array}{c}\text { Temporal_Inf_ } \\
\text { L }\end{array}$ & 89 & $-57,-21,-27$ & -4.2989 & 43 \\
\hline & & Frontal_Inf_R & 16 & $51,39,0$ & -4.4814 & 44 \\
\hline
\end{tabular}

Thresholds were set at a corrected $\mathrm{p}<0.01$ corrected by FDR criterion. BA, Brodmann's area; MNI, Montreal Neurological Institute; L, left; R, right; t0, lung cancer patients before chemotherapy; t1, lung cancer patients after chemotherapy; HCs, healthy controls 
Fig. 4 Transition matrices of different states, which were extracted using the K-means clustering method with a cluster size of 3. A The transition matrices of different states based on the right DLPFC as the seed region. B The transition matrices of different states based on the left DLPFC as the seed region

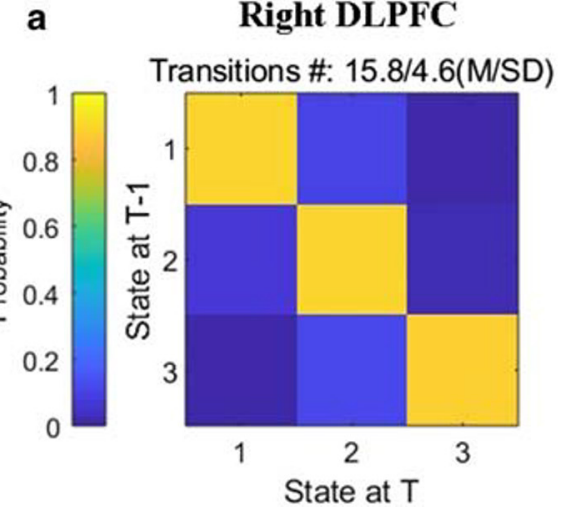

Previous studies have demonstrated altered brain sFC patterns in the ECN induced by cancer and chemotherapy. Recent evidence has shown that exploiting the dynamic properties of FC instead of traditional sFC metrics can open up novel thinking for interpreting brain functioning on different timescales (Liegeois et al. 2019). Shelli R. Kesler and his colleagues (Kesler et al. 2017) demonstrated that functional dynamics were significantly lower in patients with breast cancer than in control

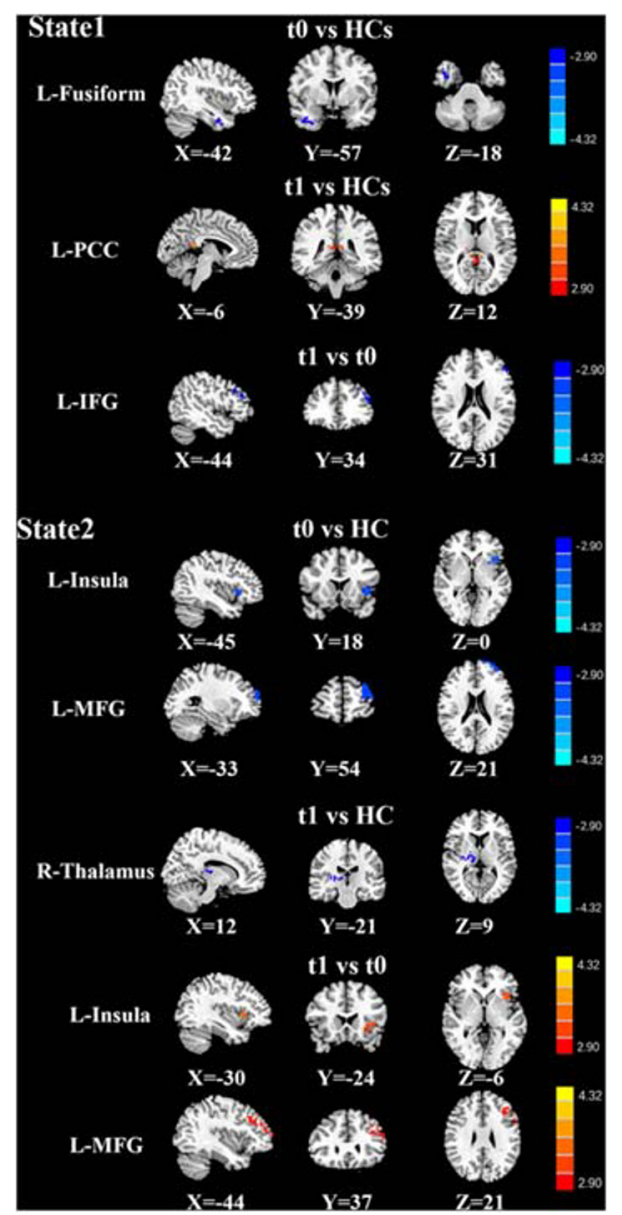

Fig. 5 Dynamic FC pattern based on the right DLPFC in each state, where lung cancer patients had a reduced or increased FC pattern in comparison to healthy controls. Significance thresholds were corrected using an FDR criterion and set at $\mathrm{p}<0.01$ participants. In this study, dFC provided additional information that was different from but complementary to the information for sFC. We found significantly decreased dFC variability between the bilateral DLPFC and right SPL and left SFG in lung cancer patients after chemotherapy and significantly reduced $\mathrm{dFC}$ between the bilateral DLPFC and left precuneus and ITG when comparing before and after chemotherapy. As mentioned above, the $\mathrm{dFC}$ variability changes after chemotherapy were consistent with the $\mathrm{sFC}$ changes.

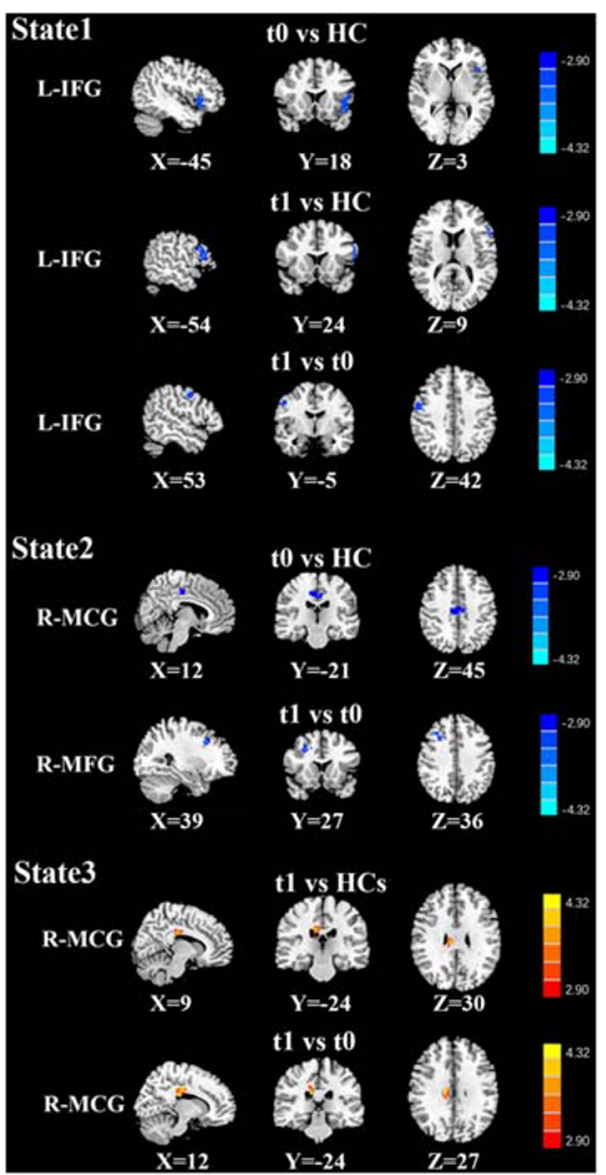

Fig. 6 Dynamic FC pattern based on the left DLPFC in each state, where lung cancer patients had a reduced or increased $\mathrm{FC}$ pattern in comparison to healthy controls. Significance thresholds were corrected using an FDR criterion and set at $\mathrm{p}<0.01$ 
Table 4 Abnormal dynamic functional connectivity of RDLPFC at different states between groups

\begin{tabular}{lllllll}
\hline States & Group comparisons & Brain region & BA & $\begin{array}{l}\text { MNI Coordinates } \\
\text { x, y, z (mm) }\end{array}$ & T score & Voxels \\
\hline State 1 & t0 vs. HCs & Fusiform_L & 55 & $-42,-57,-18$ & -2.8578 & 45 \\
& t1 vs. HCs & Cingulum_Post_ & 35 & $-6,-39,12$ & 3.2144 & 42 \\
& t1 vs. t0 & Frontal_Sup_L & 3 & $-48,36,21$ & -4.8199 & 58 \\
State 2 & t0 vs. HCs & Insula_L & 30 & $-45,18,0$ & -3.5169 & 62 \\
& & Frontal_Mid_L & 7 & $-33,54,21$ & -3.2704 & 106 \\
& t1 vs. HCs & Thalamus_R & 78 & $12,-21,9$ & -3.4867 & 66 \\
& t1 vs. t0 & Insula_L & 29 & $-30,24,-6$ & 3.9534 & 61 \\
& & Frontal_Mid_L & 7 & $-42,42,24$ & 4.4502 & 193 \\
\hline
\end{tabular}

Thresholds were set at a corrected $\mathrm{p}<0.01$ corrected by FDR criterion. BA, Brodmann's area; MNI, Montreal Neurological Institute; L, left; R, right; $\mathrm{t} 0$, lung cancer patients before chemotherapy; $\mathrm{t}$, lung cancer patients after chemotherapy; HCs, healthy controls
Interestingly, we found some significant brain FC patterns that were related to chemotherapy only when using $\mathrm{dFC}$ analysis, while $\mathrm{SFC}$ analysis did not reveal any significant correlations. For example, increased $\mathrm{dFC}$ variability between the bilateral DLPFC and left precuneus was observed before chemotherapy, and decreased dFC between the right DLPFC and left precuneus was found after chemotherapy. The precuneus, which is part of the posteromedial parietal lobe, is involved in attentive tracking, visuospatial imagination, and spatially guided behaviors (Cavanna and Trimble 2006). Simo et al. (2018) revealed that lung cancer patients after chemotherapy showed decreased FC in the left cuneus and precuneus compared to HCs. Additionally, Wang et al. (2016) found increased $\mathrm{SFC}$ between the right DLPFC and the right precuneus after chemotherapy. We hypothesize that dFC analysis seems to capture more complex FC changes than $\mathrm{sFC}$, which is also supported by the links that have been drawn between FC and the underlying brain structural connectivity.

In addition, after clustering analysis, we found that the $\mathrm{dFC}$ patterns were completely different under various connectivity states and increased dFC was even found in some states after chemotherapy. For example, in state $3, \mathrm{dFC}$ was significantly increased between the left DLPFC and right MCG. However, we could not discover these FC pattern changes during sFC analysis. Fiorenzato et al. (Fiorenzato et al. 2019) also revealed two entirely different connectivity states in Parkinson's disease patients. The cingulate cortex is usually perceived to be part of the limbic cortex, which lies immediately above the corpus callosum, and the MCG has been termed the cingulate motor area and can be activated by pain and errors made in many tasks (Kolling et al. 2016; Rolls 2019). Our results demonstrated that $\mathrm{dFC}$ analysis could capture new and latent changes in FC and that these changes were possibly derived from the dynamics of connectivity states. Even more importantly, these aforementioned findings provide evidence of increased MCG activity in lung cancer patients and allow us to speculate that this activity is a kind of compensatory mechanism in the brain activated when responding to cancer and chemotherapy.

Moreover, significant between-group differences in the temporal properties of $\mathrm{dFC}$ states were recognized in several states. Our results showed that lung cancer patients after chemotherapy tended to have a shortened MDT in certain states and a reduced NT between states, which have not been
Table 5 Abnormal dynamic functional connectivity of LDLPFC at different states between groups

\begin{tabular}{lllllll}
\hline State & Group comparisons & Brain region & BA & $\begin{array}{l}\text { MNI Coordinates } \\
\text { x, y, z (mm) }\end{array}$ & T score & Voxels \\
\hline State 1 & t0 vs. HCs & Frontal_Inf_L & 15 & $-45,18,3$ & -3.5752 & 85 \\
& t1 vs. HCs & Frontal_Inf_L & 13 & $-54,24,9$ & -2.6263 & 50 \\
& t1 vs. t0 & Frontal_Sup_R & 6 & $24,69,-3$ & -3.9716 & 71 \\
State 2 & t0 vs. HCs & Cingulum_Mid_R & 34 & $12,-21,45$ & -3.6457 & 54 \\
& t1 vs. t0 & Frontal_Mid_R & 8 & $39,27,36$ & -4.1514 & 45 \\
State 3 & t1 vs. HCs & Cingulum_Mid_R & 34 & $9,24,30$ & 3.5843 & 56 \\
& t1 vs. t0 & Cingulum_Mid_R & 34 & $12,-24,27$ & 3.6361 & 60 \\
\hline
\end{tabular}

Thresholds were set at a corrected $\mathrm{p}<0.01$ corrected by FDR criterion. BA, Brodmann's area; MNI, Montreal Neurological Institute; L, left; R, right; t0, lung cancer patients before chemotherapy; $t$, lung cancer patients after chemotherapy; HCs, healthy controls 
Fig. 7 Temporal properties of $\mathrm{dFC}$ states in the lung cancer patient and healthy control groups. A and C Mean dwell time; $\mathbf{B}$ and $\mathbf{D}$ number of transitions between states. HCs: healthy controls; t0: lung cancer patients before chemotherapy; $\mathrm{t} 1$ : lung cancer patients after chemotherapy
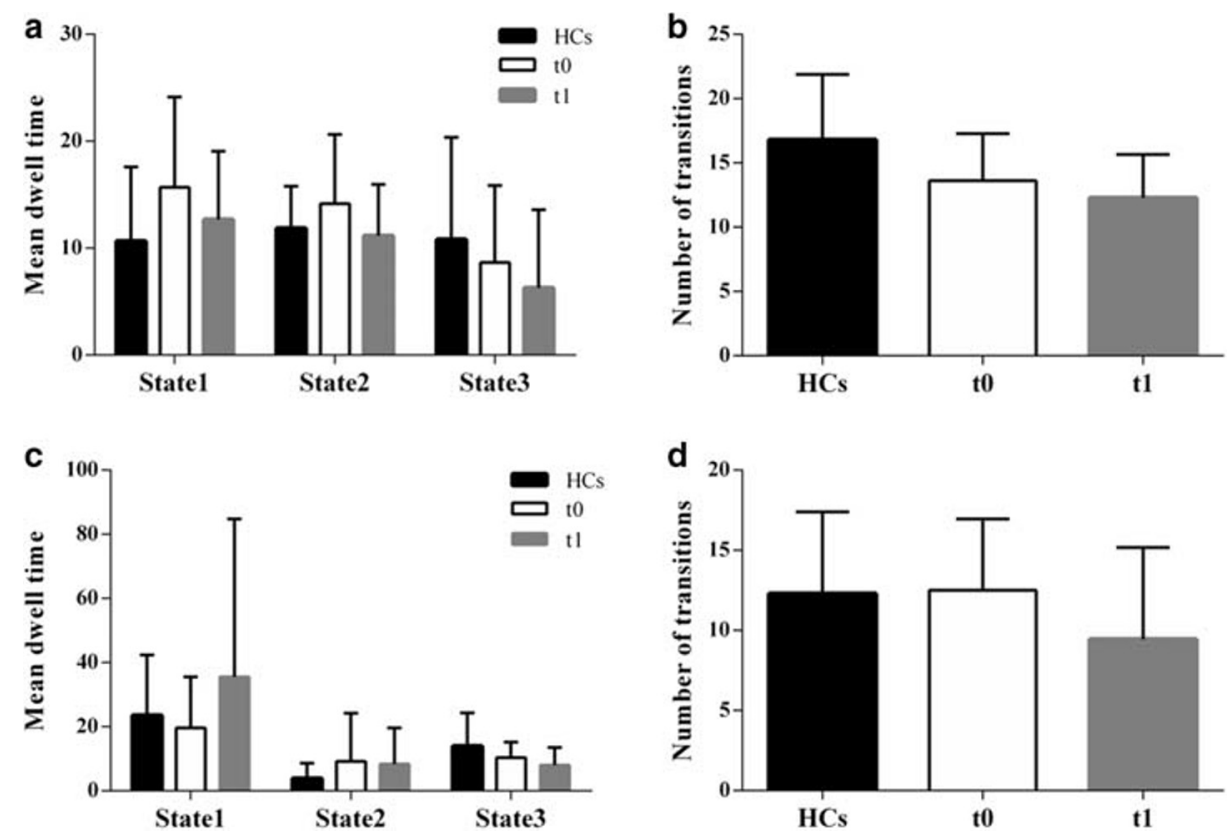

mentioned in previous studies. However, Fiorenzato et al. (Fiorenzato et al. 2019) found that an extended MDT in the segregated state and a reduced NT between states in Parkinson's disease. Moreover, as we all know that the lung cancer patients are generally treated with platinum-based chemotherapy, which is related to increased cell death and decreased cell division in the central nervous system (Dietrich et al. 2006). Therefore, the destruction of neural structures in lung cancer patients receiving platinum-based chemotherapy is likely a reason for the decreases in the MDT and NT. Thus, we may reasonably conjecture that the MDT and NT could be reference indexes to assess the effects of chemotherapy on human-brain FC pattern. Hence, more comprehensive forward-looking studies are desperately needed to explore whether the MDT and NT can be appropriate potential biomarkers of cognitive impairment in lung cancer patients.

In this study, the MoCA scores of lung cancer patients after chemotherapy were dramatically reduced. Concerning the correlations between the FC and MoCA scores, we found no significant correlations between MoCA scores and sFC, while

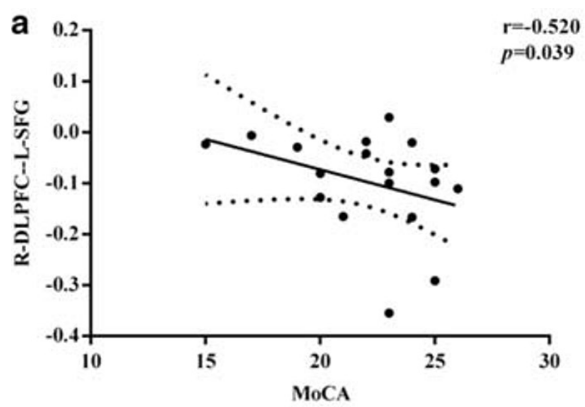

Fig. 8 A Negative correlation between the decreased dFC between the right DLPFC and left SFG and MoCA scores in patients after chemotherapy compared with nonchemotherapy patients $(r=-0.520$, negative correlations between MoCA scores and altered dFC were observed in some brain regions. Static measures of FC, which provide a measure of brain function averaged over several minutes, are oversimplified, and dynamic FC measures that capture temporal changes in brain function on the order of a few seconds have been proposed. Therefore, we judged that $\mathrm{dFC}$ could capture more information and was more sensitive for assessing brain function and cognitive defects in lung cancer patients after chemotherapy than $\mathrm{sFC}$.

There were a few limitations in this study. First, the relatively small sample size might reduce the generalizability of our results to some extent. Second, although the present study employed a longitudinal experimental design, follow-up data were collected only for patients in the lung cancer group and not for healthy controls. Previous studies (Feng et al. 2019; Pergolizzi et al. 2019) have provided evidence that cancer patients after chemotherapy exhibit FC changes during short-term follow-up (3-6 months) and have no significant difference in healthy controls. Nevertheless, all enrolled subjects should be strictly followed in subsequent longitudinal

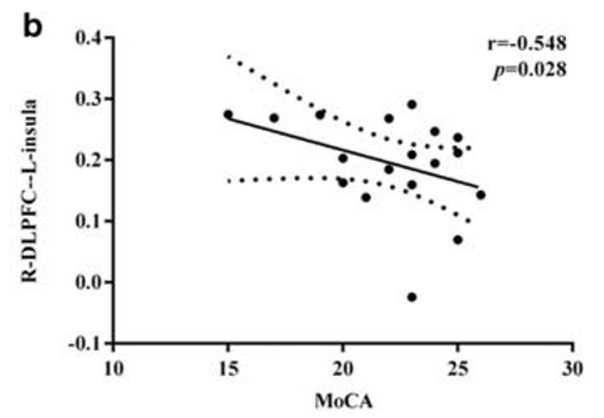

$\mathrm{p}=0.039$ ). B Negative correlation between the reduced $\mathrm{dFC}$ between the right DLPFC and left insula and MoCA scores in prechemotherapy patients compared with healthy controls $(r=-0.548, p=0.028)$ 
studies. Finally, we selected the bilateral DLPFC as the seed regions to investigate the $\mathrm{sFC}$ and $\mathrm{dFC}$ patterns in the $\mathrm{ECN}$ after chemotherapy and explore the correlational relationships with MoCA scores. The MoCA is a brief cognitive screening tool that is not very sensitive to certain domains, such as executive functions and memory, which have been exposed to be damaged after chemotherapy in previous studies. Thus, a more sophisticated and niche-targeted cognitive evaluation should be performed in future studies.

In conclusion, this is the first study to explore the $\mathrm{sFC}$ and $\mathrm{dFC}$ variations within the ECN and the relationships between the aberrant FC and cognitive decline in lung cancer patients before and after chemotherapy. Despite the limitations of the present study, our results revealed that using a dynamic connectivity analysis was more effective and sensitive for linking brain FC patterns and chemotherapy than methods that assume static brain states. The most important implication from our study is to bring awareness to cancer clinicians so they can provide information on expected CRCI to cancer patients. Moreover, several treatments are underway to help combat the symptoms of chemobrain. We concluded that the temporal dynamics of FC could be a potential biomarker to detect cognitive alterations in lung cancer patients receiving chemotherapy and provide a solid evidence for multidisciplinary rehabilitation prevention and treatment of chemobrain.

Funding information This work was supported by a grant from the National Natural Science Foundation of China (No. 81601477), Jiangsu Provincial Special Program of Medical Science (No. BE2017614), Youth Medical Talents of Jiangsu Province (No. QNRC2016062), 14th "Six Talent Peaks" Project of Jiangsu Province (No. YY-079), and Nanjing Outstanding Youth Fund (No. JQX17006).

\section{Compliance with ethical standards}

Conflict of interest The authors declare that there is no potential conflict of interests regarding the publication of this paper.

Ethical approval The current study was approved by the Research Ethics Committee of the Nanjing Medical University.

Informed consent Informed consent was obtained from all individual participants included in the study.

Open Access This article is licensed under a Creative Commons Attribution 4.0 International License, which permits use, sharing, adaptation, distribution and reproduction in any medium or format, as long as you give appropriate credit to the original author(s) and the source, provide a link to the Creative Commons licence, and indicate if changes were made. The images or other third party material in this article are included in the article's Creative Commons licence, unless indicated otherwise in a credit line to the material. If material is not included in the article's Creative Commons licence and your intended use is not permitted by statutory regulation or exceeds the permitted use, you will need to obtain permission directly from the copyright holder. To view a copy of this licence, visit http://creativecommons.org/licenses/by/4.0/.

\section{References}

Aggarwal, C., Hinneburg, A., \& Keim, D. (2001). On the surprising behavior of distance metrics in high dimensional space. Database Theory ICDT. 420-434.

Allen, E. A., Damaraju, E., Plis, S. M. et al. (2014). Tracking whole-brain connectivity dynamics in the resting state. Cerebral Cortex, 24(3), 663-676. https://doi.org/10.1093/cercor/bhs352

Baddeley, A. (2000). The episodic buffer: a new component of working memory? Trends in Cognitive Sciences, 4(11), 417-423.

Barbey, A. K., Koenigs, M., \& Grafman, J. (2013). Dorsolateral prefrontal contributions to human working memory. Cortex; a journal devoted to the study of the nervous system and behavior, 49(5), 11951205. https://doi.org/10.1016/j.cortex.2012.05.022

Bockova, M., Chladek, J., Jurak, P., Halamek, J., \& Rektor, I. (2007). Executive functions processed in the frontal and lateral temporal cortices: intracerebral study. Clinical Neurophysiology, 118(12), 2625-2636. https://doi.org/10.1016/j.clinph.2007.07.025

Bray, F., Ferlay, J., Soerjomataram, I., Siegel, R. L., Torre, L. A., \& Jemal, A. (2018). Global cancer statistics 2018: GLOBOCAN estimates of incidence and mortality worldwide for 36 cancers in 185 countries. CA: a Cancer Journal for Clinicians, 68(6), 394-424. https://doi. org/10.3322/caac. 21492

Bressler, S. L., \& Menon, V. (2010). Large-scale brain networks in cognition: emerging methods and principles. Trends in Cognitive Sciences, 14(6), 277-290. https://doi.org/10.1016/j.tics.2010.04. 004

Bromis, K., Gkiatis, K., Karanasiou, I., Matsopoulos, G., Karavasilis, E., Papathanasiou, M., et al. (2017). Altered brain functional connectivity in small-cell lung cancer patients after chemotherapy treatment: a resting-state fMRI study. Computational and Mathematical Methods in Medicine 2017, 1403940. https://doi.org/10.1155/ 2017/1403940.

Cavanna, A. E., \& Trimble, M. R. (2006). The precuneus: a review of its functional anatomy and behavioural correlates. Brain, $129(\mathrm{Pt} \mathrm{3})$, 564-583. https://doi.org/10.1093/brain/aw1004

Chen, H. J., Lin, H. L., Chen, Q. F., \& Liu, P. F. (2017). Altered dynamic functional connectivity in the default mode network in patients with cirrhosis and minimal hepatic encephalopathy. Neuroradiology, 59(9), 905-914. https://doi.org/10.1007/s00234-017-1881-4

Chen, J., Sun, D., Shi, Y., Jin, W., Wang, Y., Xi, Q. et al. (2018). Alterations of static functional connectivity and dynamic functional connectivity in motor execution regions after stroke. Neuroscience Letters, 686, 112-121. https://doi.org/10.1016/j.neulet.2018.09.008

Dietrich, J., Han, R., Yang, Y., Mayer-Proschel, M., \& Noble, M. (2006). CNS progenitor cells and oligodendrocytes are targets of chemotherapeutic agents in vitro and in vivo. Journal of Biology, 5(7), 22. https://doi.org/10.1186/jbiol50

Ettinger, D. S., Wood, D. E., Aisner, D. L., Akerley, W., Bauman, J., Chirieac, L. R. et al. (2017). Non-small cell lung cancer, version 5.2017 , NCCN clinical practice guidelines in oncology. Journal of the National Comprehensive Cancer Network, 15(4), 504-535. https://doi.org/10.6004/jncen.2017.0050

Feng, Y., Tuluhong, D., Shi, Z., Zheng, L. J., Chen, T., Lu, G. M. et al. (2019). Postchemotherapy hippocampal functional connectivity patterns in patients with breast cancer: a longitudinal resting state functional MR imaging study. Brain Imaging and Behavior. https://doi. org/10.1007/s11682-019-00067-x

Fiorenzato, E., Strafella, A. P., Kim, J., Schifano, R., Weis, L., Antonini, A. et al. (2019). Dynamic functional connectivity changes associated with dementia in Parkinson's disease. Brain, 142(9), 2860-2872. https://doi.org/10.1093/brain/awz192

Fu, Z., Tu, Y., Di, X., Du, Y., Pearlson, G. D., Turner, J. A. et al. (2018). Characterizing dynamic amplitude of low-frequency fluctuation and its relationship with dynamic functional connectivity: an application 
to schizophrenia. Neuroimage, 180(Pt B), 619-631. https://doi.org/ 10.1016/j.neuroimage.2017.09.035

Funahashi, S., \& Andreau, J. M. (2013). Prefrontal cortex and neural mechanisms of executive function. Journal of Physiology, Paris, 107(6), 471-482. https://doi.org/10.1016/j.jphysparis.2013.05.001

Galea, M., \& Woodward, M. (2005). Mini-Mental State Examination (MMSE). The Australian Journal of Physiotherapy, 51(3), 198.

Gonzalez-Castillo, J., Hoy, C. W., Handwerker, D. A., Robinson, M. E., Buchanan, L. C., Saad, Z. S. et al. (2015). Tracking ongoing cognition in individuals using brief, whole-brain functional connectivity patterns. Proceedings of the National Academy of Sciences of the United States of America, 112(28), 8762-8767. https://doi.org/10. 1073/pnas. 1501242112

Grosshans, D. R., Meyers, C. A., Allen, P. K., Davenport, S. D., \& Komaki, R. (2008). Neurocognitive function in patients with small cell lung cancer: effect of prophylactic cranial irradiation. Cancer, 112(3), 589-595. https://doi.org/10.1002/cncr.23222

Hutchison, R. M., Womelsdorf, T., Gati, J. S., Everling, S., \& Menon, R. S. (2013). Resting-state networks show dynamic functional connectivity in awake humans and anesthetized macaques. Human Brain Mapping, 34(9), 2154-2177. https://doi.org/10.1002/hbm.22058

Janelsins, M. C., Kohli, S., Mohile, S. G., Usuki, K., Ahles, T. A., \& Morrow, G. R. (2011). An update on cancer- and chemotherapyrelated cognitive dysfunction: current status. Seminars in Oncology, 38(3), 431-438. https://doi.org/10.1053/j.seminoncol. 2011.03.014

Janelsins, M. C., Kesler, S. R., Ahles, T. A., \& Morrow, G. R. (2014). Prevalence, mechanisms, and management of cancer-related cognitive impairment. International Review of Psychiatry, 26(1), 102113. https://doi.org/10.3109/09540261.2013.864260

Janelsins, M. C., Heckler, C. E., Peppone, L. J., Ahles, T. A., Mohile, S. G., Mustian, K. M. et al. (2018). Longitudinal trajectory and characterization of cancer-related cognitive impairment in a nationwide cohort study. Journal of Clinical Oncology. Jco2018786624. https:// doi.org/10.1200/jco.2018.78.6624

Jodzio, K., \& Biechowska, D. (2010). Wisconsin card sorting test as a measure of executive function impairments in stroke patients. Applied Neuropsychology, 17(4), 267-277. https://doi.org/10.1080/ 09084282.2010 .525104

Kaasa, S., Olsnes, B. T., Thorud, E., \& Host, H. (1988). Reduced shortterm neuropsychological performance in patients with nonsmall-cell lung cancer treated with cisplatin and etoposide. Antibiotics and Chemotherapy (1971), 41(41), 226-231.

Kesler, S. R., Kent, J. S., \& O'Hara, R. (2011). Prefrontal cortex and executive function impairments in primary breast cancer. Archives of Neurology, 68(11), 1447-1453. https://doi.org/10.1001/ archneurol.2011.245

Kesler, S. R., Adams, M., Packer, M., Rao, V., Henneghan, A. M., Blayney, D. W., et al. (2017). Disrupted brain network functional dynamics and hyper-correlation of structural and functional connectome topology in patients with breast cancer prior to treatment. Brain and Behavior: A Cognitive Neuroscience Perspective 7(3), e00643. https://doi.org/10.1002/brb3.643.

Kolling, N., Wittmann, M. K., Behrens, T. E., Boorman, E. D., Mars, R. B., \& Rushworth, M. F. (2016). Value, search, persistence and model updating in anterior cingulate cortex. Nature Neuroscience, 19(10), 1280-1285. https://doi.org/10.1038/nn.4382

Leonardi, N., \& Van De Ville, D. (2015). On spurious and real fluctuations of dynamic functional connectivity during rest. Neuroimage, 104, 430-436. https://doi.org/10.1016/j.neuroimage.2014.09.007.

Li, M., \& Caeyenberghs, K. (2018). Longitudinal assessment of chemotherapy-induced changes in brain and cognitive functioning: A systematic review. Neuroscience and Biobehavioral Reviews, 92, 304-317. https://doi.org/10.1016/j.neubiorev.2018.05.019

Liao, W., Wu, G. R., Xu, Q., Ji, G. J., Zhang, Z., Zang, Y. F. et al. (2014). DynamicBC: a MATLAB toolbox for dynamic brain connectome analysis. Brain Connectivity, 4(10), 780-790. https://doi.org/10. 1089/brain.2014.0253

Liao, W., Li, J., Duan, X., Cui, Q., Chen, H., \& Chen, H. (2018). Static and dynamic connectomics differentiate between depressed patients with and without suicidal ideation. Human Brain Mapping, 39(10), 4105-4118. https://doi.org/10.1002/hbm.24235

Liegeois, R., Li, J., Kong, R., Orban, C., Van De Ville, D., Ge, T. et al. (2019). Resting brain dynamics at different timescales capture distinct aspects of human behavior. Nature Communications, 10(1), 2317. https://doi.org/10.1038/s41467-019-10317-7

Lowe, M. J., Mock, B. J., \& Sorenson, J. A. (1998). Functional connectivity in single and multislice echoplanar imaging using resting-state fluctuations. Neuroimage, 7(2), 119-132. https://doi.org/10.1006/ nimg. 1997.0315

Maldjian, J. A., Laurienti, P. J., Kraft, R. A., \& Burdette, J. H. (2003). An automated method for neuroanatomic and cytoarchitectonic atlasbased interrogation of fMRI data sets. Neuroimage, 19(3), 12331239.

Mandelblatt, J. S., Hurria, A., McDonald, B. C., Saykin, A. J., Stern, R. A., VanMeter, J. W. et al. (2013). Cognitive effects of cancer and its treatments at the intersection of aging: what do we know; what do we need to know? Seminars in Oncology, 40(6), 709-725. https:// doi.org/10.1053/j.seminoncol.2013.09.006

McDonald, B. C., Conroy, S. K., Ahles, T. A., West, J. D., \& Saykin, A. J. (2012). Alterations in brain activation during working memory processing associated with breast cancer and treatment: a prospective functional magnetic resonance imaging study. Journal of Clinical Oncology, 30(20), 2500-2508. https://doi.org/10.1200/jco.2011.38. 5674

Menning, S., de Ruiter, M. B., Veltman, D. J., Boogerd, W., Oldenburg, H. S., Reneman, L., et al. (2017). Changes in brain activation in breast cancer patients depend on cognitive domain and treatment type. PLoS One 12(3), e0171724. https://doi.org/10.1371/journal. pone. 0171724

Mitchell, D. G., Rhodes, R. A., Pine, D. S., \& Blair, R. J. (2008). The contribution of ventrolateral and dorsolateral prefrontal cortex to response reversal. Behavioural Brain Research, 187(1), 80-87. https://doi.org/10.1016/j.bbr.2007.08.034

Pergolizzi, D., Root, J. C., Pan, H., Silbersweig, D., Stern, E., Passik, S. D., et al. (2019). Episodic memory for visual scenes suggests compensatory brain activity in breast cancer patients: a prospective longitudinal fMRI study. Brain Imaging and Behavior. https://doi.org/ 10.1007/s11682-019-00038-2

Piccirillo, J. F., Hardin, F. M., Nicklaus, J., Kallogjeri, D., Wilson, M., Ma, C. X. et al. (2015). Cognitive impairment after chemotherapy related to atypical network architecture for executive control. Oncology (Williston Park, N.Y.), 88(6), 360-368. https://doi.org/ 10.1159/000370117

Pochon, J. B., Levy, R., Poline, J. B., Crozier, S., Lehericy, S., Pillon, B. et al. (2001). The role of dorsolateral prefrontal cortex in the preparation of forthcoming actions: an fMRI study. Cerebral Cortex, 11(3), 260-266. https://doi.org/10.1093/cercor/11.3.260

Rolls, E. T. (2019). The cingulate cortex and limbic systems for emotion, action, and memory. Brain Structure \& Function. https://doi.org/10. 1007/s00429-019-01945-2.

Rosazza, C., \& Minati, L. (2011). Resting-state brain networks: literature review and clinical applications. Neurological Sciences, 32(5), 773785. https://doi.org/10.1007/s10072-011-0636-y

Royall, D. R., Lauterbach, E. C., Cummings, J. L., Reeve, A., Rummans, T. A., Kaufer, D. I. et al. (2002). Executive control function: a review of its promise and challenges for clinical research. A report from the Committee on Research of the American Neuropsychiatric Association. The Journal of Neuropsychiatry and Clinical Neurosciences, 14(4), 377-405. https://doi.org/10.1176/jnp.14.4. 377 
Rusnakova, S., Daniel, P., Chladek, J., Jurak, P., \& Rektor, I. (2011). The executive functions in frontal and temporal lobes: a flanker task intracerebral recording study. Journal of Clinical Neurophysiology, 28(1), 30-35. https://doi.org/10.1097/WNP.0b013e31820512d4

Scagliotti, G. V., Parikh, P., von Pawel, J., Biesma, B., Vansteenkiste, J., Manegold, C. et al. (2008). Phase III study comparing cisplatin plus gemcitabine with cisplatin plus pemetrexed in chemotherapy-naive patients with advanced-stage non-small-cell lung cancer. Journal of Clinical Oncology, 26(21), 3543-3551. https://doi.org/10.1200/jco. 2007.15.0375

Shirer, W. R., Ryali, S., Rykhlevskaia, E., Menon, V., \& Greicius, M. D. (2012). Decoding subject-driven cognitive states with whole-brain connectivity patterns. Cerebral Cortex, 22(1), 158-165. https://doi. org/10.1093/cercor/bhr099

Simo, M., Root, J. C., Vaquero, L., Ripolles, P., Jove, J., Ahles, T. et al. (2015). Cognitive and brain structural changes in a lung cancer population. Journal of Thoracic Oncology, 10(1), 38-45. https:// doi.org/10.1097/jto.0000000000000345

Simo, M., Vaquero, L., Ripolles, P., Gurtubay-Antolin, A., Jove, J., Navarro, A. et al. (2016). Longitudinal brain changes associated with prophylactic cranial irradiation in lung cancer. Journal of Thoracic Oncology, 11(4), 475-486. https://doi.org/10.1016/j.jtho. 2015.12.110

Simo, M., Rifa-Ros, X., Vaquero, L., Ripolles, P., Cayuela, N., Jove, J. et al. (2018). Brain functional connectivity in lung cancer population: an exploratory study. Brain Imaging and Behavior, 12(2), 369382. https://doi.org/10.1007/s11682-017-9697-8

Thompson, G. J., Magnuson, M. E., Merritt, M. D., Schwarb, H., Pan, W. J., McKinley, A. et al. (2013). Short-time windows of correlation between large-scale functional brain networks predict vigilance intraindividually and interindividually. Human Brain Mapping, 34(12), 3280-3298. https://doi.org/10.1002/hbm.22140

van den Heuvel, M., Mandl, R., \& Hulshoff Pol, H. (2008). Normalized cut group clustering of resting-state FMRI data. PLoS One, 3(4), e2001. https://doi.org/10.1371/journal.pone.0002001.

Van Dijk, K. R., Hedden, T., Venkataraman, A., Evans, K. C., Lazar, S. W., \& Buckner, R. L. (2010). Intrinsic functional connectivity as a tool for human connectomics: theory, properties, and optimization. Journal of Neurophysiology, 103(1), 297-321. https://doi.org/10. 1152/jn.00783.2009

Wang, J., Wang, X., Xia, M., Liao, X., Evans, A., \& He, Y. (2015). GRETNA: a graph theoretical network analysis toolbox for imaging connectomics. Frontiers in Human Neuroscience, 9, 386. https:// doi.org/10.3389/fnhum.2015.00386

Wang, L., Yan, Y., Wang, X., Tao, L., Chen, Q., Bian, Y. et al. (2016). Executive function alternations of breast cancer patients after chemotherapy: evidence from resting-state functional MRI. Academic Radiology, 23(10), 1264-1270. https://doi.org/10.1016/j.acra.2016. 05.014

Weiland, B. J., Sabbineni, A., Calhoun, V. D., Welsh, R. C., Bryan, A. D., Jung, R. E. et al. (2014). Reduced left executive control network functional connectivity is associated with alcohol use disorders. Alcoholism, Clinical and Experimental Research, 38(9), 24452453. https://doi.org/10.1111/acer.12505

Welzel, T., Niethammer, A., Mende, U., Heiland, S., Wenz, F., Debus, J. et al. (2008). Diffusion tensor imaging screening of radiationinduced changes in the white matter after prophylactic cranial irradiation of patients with small cell lung cancer: first results of a prospective study. AJNR. American Journal of Neuroradiology, 29(2), 379-383. https://doi.org/10.3174/ajnr.A0797

Yang, S., Meng, Y., Li, J., Fan, Y. S., Du, L., Chen, H. et al. (2019). Temporal dynamic changes of intrinsic brain activity in schizophrenia with cigarette smoking. Schizophrenia Research, 210, 66-72. https://doi.org/10.1016/j.schres.2019.06.012

Zhang, Y., Chen, Y. C., Hu, L., You, J., Gu, W., Li, Q., et al. (2019). Chemotherapy-induced functional changes of the default mode network in patients with lung cancer. Brain Imaging and Behavior. https://doi.org/10.1007/s11682-018-0030-y.

Publisher's note Springer Nature remains neutral with regard to jurisdictional claims in published maps and institutional affiliations. 